\title{
The hidden duplication past of the plant pathogen Phytophthora and its consequences for infection
}

\author{
Cindy Martens ${ }^{1,2}$ and Yves Van de Peer*1,2
}

\begin{abstract}
Background: Oomycetes of the genus Phytophthora are pathogens that infect a wide range of plant species. For dicot hosts such as tomato, potato and soybean, Phytophthora is even the most important pathogen. Previous analyses of Phytophthora genomes uncovered many genes, large gene families and large genome sizes that can partially be explained by significant repeat expansion patterns.

Results: Analysis of the complete genomes of three different Phytophthora species, using a newly developed approach, unveiled a large number of small duplicated blocks, mainly consisting of two or three consecutive genes. Further analysis of these duplicated genes and comparison with the known gene and genome duplication history of ten other eukaryotes including parasites, algae, plants, fungi, vertebrates and invertebrates, suggests that the ancestor of P. infestans, P. sojae and P. ramorum most likely underwent a whole genome duplication (WGD). Genes that have survived in duplicate are mainly genes that are known to be preferentially retained following WGDs, but also genes important for pathogenicity and infection of the different hosts seem to have been retained in excess. As a result, the WGD might have contributed to the evolutionary and pathogenic success of Phytophthora.

Conclusions: The fact that we find many small blocks of duplicated genes indicates that the genomes of Phytophthora species have been heavily rearranged following the WGD. Most likely, the high repeat content in these genomes have played an important role in this rearrangement process. As a consequence, the paucity of retained larger duplicated blocks has greatly complicated previous attempts to detect remnants of a large-scale duplication event in Phytophthora. However, as we show here, our newly developed strategy to identify very small duplicated blocks might be a useful approach to uncover ancient polyploidy events, in particular for heavily rearranged genomes.
\end{abstract}

\section{Background}

Oomycetes or water molds form a diverse group of eukaryotic micro-organisms that have originally been classified as Fungi because of their similarity in growth morphology, propagation through spores and weaponry to infect host organisms [1]. Furthermore, they occupy similar ecological niches and share many cell wall degrading enzymes to weaken host tissues [2,3]. However, biochemical and molecular data have shown that oomycetes have little affinity with "true" fungi but are instead more closely related to heterokont algae and diatoms [4,5], belonging to the assemblage chromalveolates, which also include organisms such as ciliates, apicomplexans and

\footnotetext{
*Correspondence: yves.vandepeer@psb.vib-ugent.be

1 Department of Plant Systems Biology, VIB, Technologiepark 927, B-9052 Ghent, Belgium

Full list of author information is available at the end of the article
}

dinoflagellates [6,7]. Also in contrast to fungi, oomycetes are diploid organisms that lack a free haploid life stage.

Members of the genus Phytophthora cause devastating diseases on a wide range of plants, and are the most important pathogens of dicots. For instance, Phytophthora infestans, responsible for severe damage in the food production worldwide by infecting tomato and potato [8], was the infective agent of the so-called potato blight that caused the Irish famine between 1845 and 1849 , during which approximately one million people died and another million emigrated $[9,10]$. Another species, P. sojae, causes root and stem rot in soybean resulting in huge annual production losses [11].

So far, three Phytophthora species have been fully sequenced and annotated, namely P. sojae, P. ramorum and P. infestans. Breakouts of the 'sudden oak death' disease caused by $P$. ramorum led to the first Phytophthora genome project. Since there were no close relatives 
sequenced yet, a second genome, the one of Phytophthora sojae, was sequenced simultaneously. $P$. sojae and $P$. ramorum have a genome size of $95 \mathrm{Mb}$ and $65 \mathrm{Mb}$, respectively [12]. $P$. infestans, of which the genome sequence has been determined recently as well, has an estimated genome size of $240 \mathrm{Mb}$ [13]. In comparison to other plant pathogens, the Phytophthora genomes are quite large. Bacterial genomes are often smaller than 10 $\mathrm{Mb}$ and fungal genomes rarely exceed $40 \mathrm{Mb}$ [14]. The larger size of the P. sojae genome compared to P. ramorum is not only because of the higher number of predicted genes (16.988 and 14.451, respectively [13]) but also because of larger intergenic regions and different retrotransposon expansion patterns $[12,15]$. In P. infestans, which has 17.797 predicted genes [13], the intergenic regions are even larger than in $P$. sojae and the number of different types of transposons is overwhelming [13,1618]. The $P$. infestans genome is by far the largest chromalveolate genome sequenced and Haas and colleagues (2009) have shown that its expansion results from a proliferation of repetitive DNA accounting for $~ 74 \%$ of the genome [13]. Comparison of the three Phytophthora genomes also revealed an unusual genome organization; i.e. regions with conserved gene order, high gene density and lower repeat content are separated by regions with non-conserved gene order, low gene density and high repeat content [13].

In a previous study, we observed that Phytophthora species have many more genes than most other chromalveolate species for which the complete genome sequence has been determined [19]. Also the average gene family size is larger than for the other chromalveolates, except for the ciliates Paramecium tetraurelia, which has undergone three whole genome duplication events [20] and Tetrahymena thermophila, which has undergone an extensive number of tandem duplications [21]. Furthermore, in particular genes important for the interaction with their hosts, such as genes encoding cell wall degrading enzymes, often seem to have been duplicated in Phytophthora species $[19,22]$. Here, we have tried to unravel the duplication past of the three Phytophthora species and conclude that many of the duplicated genes are likely the result of a shared ancient large-scale or even whole genome duplication event.

\section{Results}

\section{Duplicated regions in the Phytophthora genome}

If an organism has undergone a large-scale or whole genome duplication (WGD) in its evolutionary past, there is a reasonable chance to find remnants of this event. For instance, such remnants can be detected by the identification of genomic segments sharing a set of homologous genes [23]. When also the order of the homologous genes (sometimes referred to as anchor points or anchors) on the chromosomes is still conserved, the evidence for a block duplication is strengthened. To define homologous gene pairs within each of the Phytophthora species and reference organisms, the proteomes were grouped into gene families based on sequence similarity and Markov clustering (see Methods). Gene families with more than 100 members were omitted from the analysis since these gene families are often artefacts of the gene family clustering methods, i.e. artificial clustering of different families into superfamilies. Also gene pairs with a $\mathrm{K}_{\mathrm{S}}$-value lower than 0.1 and/or lying on a small scaffold (i.e. fewer than 6 genes) were omitted from the analysis (see Methods).

Using our previously developed software i-ADHoRe [24], we identified blocks of homologous genes in the Phytophthora genomes. In brief, the i-ADHoRe algorithm detects homologous (duplicated) regions in a genome by identifying diagonals in a gene homology matrix, after which the longest diagonal or duplicated region is reported. The whole procedure is controlled by a set of parameters including gap size, which describes the maximal number of intervening, non-homologous genes tolerated between two homologous genes within a collinear segment, and a parameter determining to what extent the elements of a cluster fit on a diagonal line. Because of its specific development and implementation, the algorithm can only detect clusters of at least three homologous gene pairs $[23,25]$.

To our surprise, the large majority of duplicated blocks in P. infestans consist of only three homologous gene pairs (only one block of five duplicated genes and seven blocks of four genes could be detected; data not shown). The same is observed for both other Phytophthora species, namely $P$. sojae and P. ramorum (data not shown). For all duplicated blocks, we also counted the number of intervening (non-homologous) genes. Strikingly, the average number of intervening genes is extremely small and in most cases the duplicated genes in these small blocks are located directly next to each other.

The fact that Phytophthora species, especially $P$. infestans and $P$. sojae, have a large number of genes and many multicopy gene families [19], as well as many duplicated blocks of three homologs, raised the question whether these blocks could be the remnants of a large-scale gene or even entire genome duplication event. Furthermore, the presence of a high number of very small duplicated blocks could point to an ancient duplication event followed by a large number of genome rearrangements breaking up larger blocks. If this were true, we would expect to find even more blocks with only two homologous genes. 


\section{Finding small duplicated blocks of genes}

Since i-ADHoRe and most other methods for duplicated block detection have been developed specifically to report large homologous segments between or within genomes [23], we developed a new approach to detect small duplicated blocks of only two or three homologous genes, referred to as $2 \mathrm{HOM}$ and $3 \mathrm{HOM}$ blocks, respectively (see Methods and Figure 1, Panel A and B). As can be seen in Figure 1 (Panel B), 2HOM blocks can be part of a $3 \mathrm{HOM}$ block, which is different from for example $\mathrm{i}$ $\mathrm{ADHoRe}$ or related software reporting only the longest blocks. Similarly, our 3HOM blocks can be part of a block with more than three homologous genes. A genome that has been recently duplicated and did not undergo extensive genome rearrangements will have almost no blocks of only two or three anchor points using the i-ADHoRe approach because most of them will be part of a larger block. However, in our new strategy such a genome will count numerous $2 \mathrm{HOM}$ and $3 \mathrm{HOM}$ blocks, indicative of a large-scale duplication event. In this respect, we can compare the distribution of $2 \mathrm{HOM}$ and $3 \mathrm{HOM}$ blocks between duplicated and non-duplicated genomes, trying to reduce the effect of genome rearrangements and the breaking up of larger duplicated blocks. It should be noted that blocks comprised of gene families with more than 100 members were discarded from all further analyses.

Because we are looking at very small duplicated blocks, the possibility of finding some of those by chance is greater than for larger blocks. Therefore, in our block detection method, we have chosen to be more stringent by not allowing any intervening genes. Moreover, to compare our observation with what could be expected by chance, we ran 1000 simulations for every genome, i.e. every genome was shuffled 1000 times and for each random genome the number of $2 \mathrm{HOM}$ and $3 \mathrm{HOM}$ blocks was counted. The results for the $2 \mathrm{HOM}$ block detection in the three different Phytophthora species are shown in Figure 2A, where the pink triangle shows the number of blocks found in the real genome, while the distributions shown in blue represent the results for random data, i.e. the output of the 1000 simulations. If the number of blocks found in the real data is clearly different from the number of blocks found in the randomizations, the number of blocks in this species is highly significant, meaning that we find more blocks than we would expect by chance alone. As can be seen in Figure 2A, the number of 2HOM blocks detected in all three Phytophthora species is significantly different from what would be expected by chance alone. The same is true for the 3HOM blocks (Figure 2B), where we can also see that $3 \mathrm{HOM}$ blocks without intervening genes are extremely rare in randomized genomes. Moreover, as expected, in all three Phytophthora genomes the number of $2 \mathrm{HOM}$ blocks is much higher than the number of $3 \mathrm{HOM}$ blocks (Table 1).

Because the number of small duplicated blocks in Phytophthora genomes seems unexpectedly high, we compared them with the number of blocks found in other genomes, which we will further refer to as the reference genomes. We distinguished three types of reference genomes, i.e. those of organisms that (i) underwent at least one WGD in their evolutionary past (Arabidopsis thaliana [26,27], Saccharomyces cerevisiae [28,29], Homo sapiens [30], and T. nigroviridis [31,32]), (ii) underwent segmental duplications (C. elegans [33-36], P. falciparum [37] and K. lactis [38,39]), (iii) most likely have not been duplicated (P. tricornutum, D. melanogaster and A. gambiae). For all organisms, we applied the same detection strategy. The results for the detection of $2 \mathrm{HOM}$ and 3HOM blocks are shown in Table 1.

Figure 3A shows the results of the $2 \mathrm{HOM}$ block simulation analyses for all genomes (for a detailed overview, see Additional file 1). Again, the colored triangles represent the number of blocks that we find in the real genomes, while the corresponding distributions represent the 1000 simulations for every genome. As can be observed, there are three clusters of organisms that can be discerned: one formed by the reference species that have not undergone large-scale gene duplication events, one by the three Phytophthora species, human and C. elegans, and one by Arabidopsis and Tetraodon. These latter two organisms show an extremely high number of $2 \mathrm{HOM}$ blocks, which is explained by the fact that both Arabidopsis and Tetraodon have undergone multiple rounds of WGDs. For instance, it has been shown recently that the Arabidopsis genome has undergone at least three WGD events, two of which have occurred during the last 70 or so my $[26,27]$. Ray-finned fishes, such as Tetraodon, have undergone a WGD about 300 mya, after their divergence from the land vertebrates $[31,32]$. On top of that, they share two rounds of earlier genome duplications with the other vertebrates [40]. So, all organisms for which it has been clearly demonstrated that they have doubled their genome during their evolutionary past show a very high number of $2 \mathrm{HOM}$ duplicated blocks. The only exception is the yeast $S$. cerevisiae, which has undergone a WGD 100 mya $[28,31]$. However, when we correct for the number of genes in the genome, this discrepancy disappears (see further).

Table 1 summarizes the results of the detection of the 3HOM blocks in the Phytophthora and reference genomes (for a detailed overview, see Additional file 2). Next to A. thaliana, $P$. infestans has the highest number of $3 \mathrm{HOM}$ blocks. The number of $3 \mathrm{HOM}$ blocks in the other two Phytophthora species is much lower, but still higher than in S. cerevisiae. 


\section{A Detection of $2 \mathrm{HOM}$ duplicated blocks}

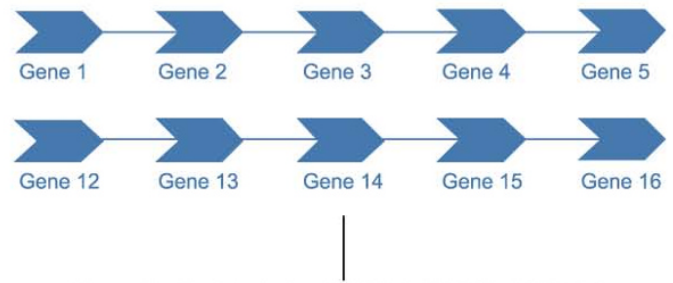

Gene Family Clustering (BLASTP, MCLBLASTLINE)

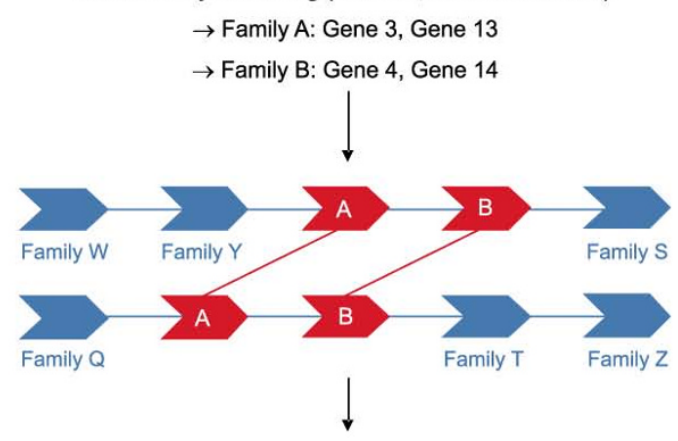

One 2HOM Block (duplicated pair AB)

\section{Conserved orientation}

a.

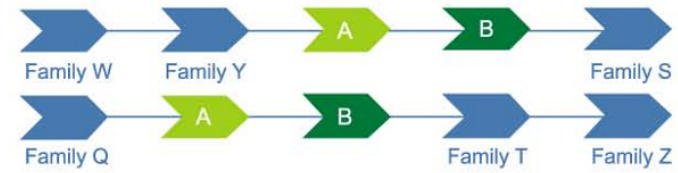

b.

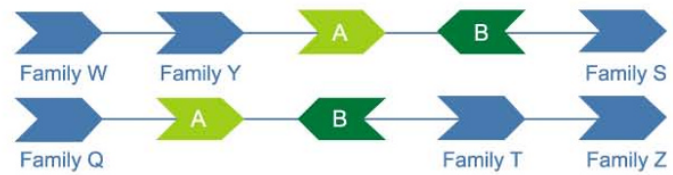

c.

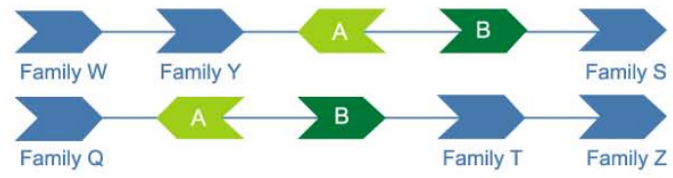

d.

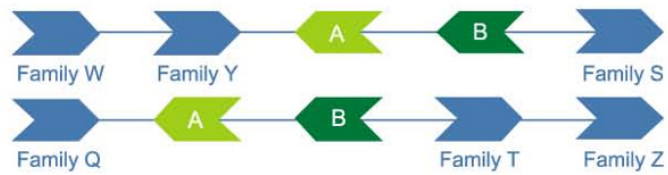

B Detection of 3HOM duplicated blocks

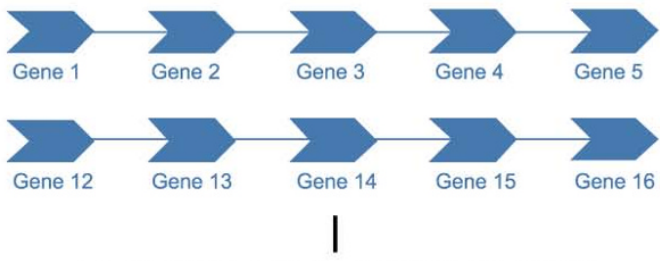

Gene Family Clustering (BLASTP, MCLBLASTLINE)

$\rightarrow$ Family A: Gene 3, Gene 13

$\rightarrow$ Family B: Gene 4, Gene 14

$\rightarrow$ Family C: Gene 5, Gene 15

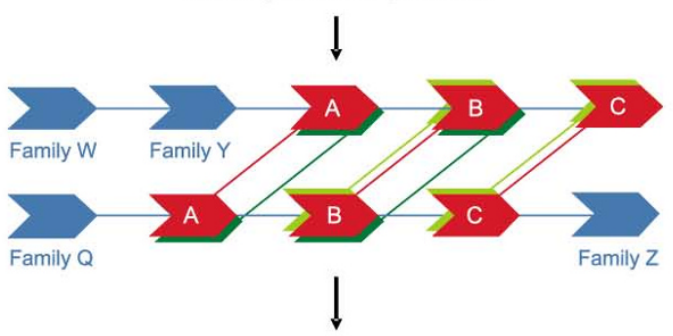

One 3HOM Block (duplicated pair $\mathrm{ABC}$ )

Two $2 \mathrm{HOM}$ Blocks (duplicated pairs $\mathrm{AB}$ and $\mathrm{BC}$ )

\section{Conserved orientation but inverted segments}

a.

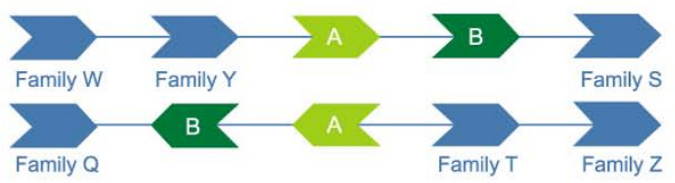

b.

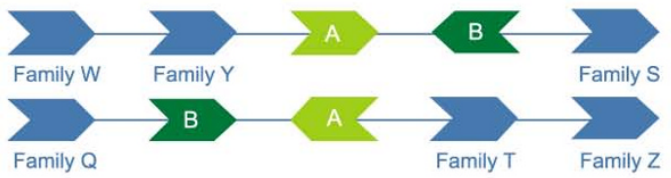

c.

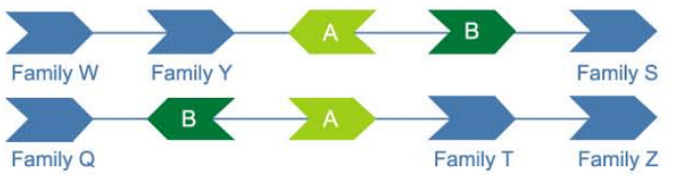

d.

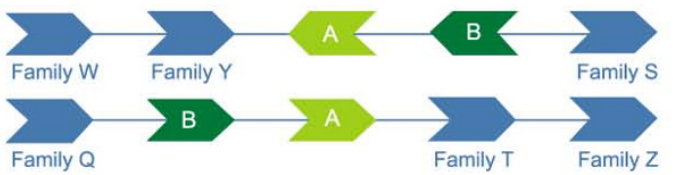

Figure 1 Detection of small duplicated genomic segments. (A) Detection of $2 \mathrm{HOM}$ blocks in real and random genomes. In red, a duplicated block of two homologous genes $A B$ is shown. (B) Detection of $3 \mathrm{HOM}$ blocks in real and random genomes. In red, a duplicated block of three homologous genes $\mathrm{ABC}$ is shown. In this example, also two $2 \mathrm{HOM}$ blocks are identified, namely AB (dark green) and BC (light green). (C) All possible situations where the gene orientation in a $2 \mathrm{HOM}$ block is conserved and not inverted. (D) Possible situations where the gene orientation in a $2 \mathrm{HOM}$ block is conserved but where one segment is inverted. 
Table 1: 2HOM and 3HOM block detection in Phytophthora and reference genomes.

\begin{tabular}{|c|c|c|c|}
\hline Organism & No. of filtered blocks & No. of possible filtered blocks & Percentage of filtered blocks \\
\hline \multicolumn{4}{|c|}{ 2HOM block detection } \\
\hline P. infestans & 253 & 11,661 & 2.17 \\
\hline P. sojae & 207 & 10,816 & 1.91 \\
\hline P. ramorum & 128 & 8,706 & 1.47 \\
\hline P. tricornutum & 24 & 9,559 & 0.25 \\
\hline P. falciparum & 18 & 4,945 & 0.36 \\
\hline S. cerevisiae & 51 & 5,516 & 0.92 \\
\hline K. lactis & 12 & 5,235 & 0.23 \\
\hline A. thaliana & 651 & 18,805 & 3.46 \\
\hline H. sapiens & 258 & 16,746 & 1.54 \\
\hline T. nigroviridis & 531 & 15,094 & 3.52 \\
\hline C. elegans & 155 & 16,224 & 0.96 \\
\hline D. melanogaster & 6 & 12,262 & 0.05 \\
\hline A. gambiae & 46 & 10,140 & 0.45 \\
\hline \multicolumn{4}{|c|}{ 3HOM block detection } \\
\hline P. infestans & 77 & 11,224 & 0.69 \\
\hline P. sojae & 15 & 10,062 & 0.15 \\
\hline P. ramorum & 12 & 8,206 & 0.15 \\
\hline P. tricornutum & 2 & 9,525 & 0.02 \\
\hline P. falciparum & 4 & 4,919 & 0.0808 \\
\hline S. cerevisiae & 8 & 5,516 & 0.15 \\
\hline K. lactis & 5 & 5,233 & 0.10 \\
\hline A. thaliana & 90 & 17,410 & 0.52 \\
\hline H. sapiens & 28 & 15,868 & 0.18 \\
\hline T. nigroviridis & 71 & 14,367 & 0.49 \\
\hline C. elegans & 25 & 15,835 & 0.16 \\
\hline D. melanogaster & 1 & 11,867 & 0.01 \\
\hline A.gambiae & 2 & 9,898 & 0.02 \\
\hline
\end{tabular}

There is no evidence that the genome of C. elegans has been duplicated. However, it has been shown that this genome has undergone segmental duplication [33-36], which explains its relatively large number of 2- and $3 \mathrm{HOM}$ blocks. To investigate whether many small blocks in the Phytophthora genomes could also be explained by a few segmental or chromosomal duplications, we calculated the percentage of genomic scaffolds containing at least one small duplicated block. After removal of large gene families, 66, 67 and $61 \%$ of the P. infestans, P. sojae and $P$. ramorum scaffolds, respectively, contain at least one block. When we count the number of scaffolds with two up to 60 duplicated blocks, the number of scaffolds in all three Phytophthora species gradually decreases when the number of detected blocks increases (see Additional file 3). Moreover, we observed that the number of blocks detected on a scaffold is linearly correlated with the size of the scaffold, expressed in the number of genes (see Additional file 4). Finally, in order to make sure that the small duplicated blocks are not operon-like structures, we considered functional clustering and intergenic distances within the duplicated blocks (see Additional file 5). The results of these analyses rejected the operon-hypothesis (see Additional file 5 and Additional file 6).

\section{Dating the block duplications}

The identification of many segmental duplications is usually considered strong evidence for a WGD, although it is hard to rule out that they are the result of many independent segmental duplications. However, if one can show 
A Number of blocks with two homologous gene pairs, without intervening genes (2HOM)
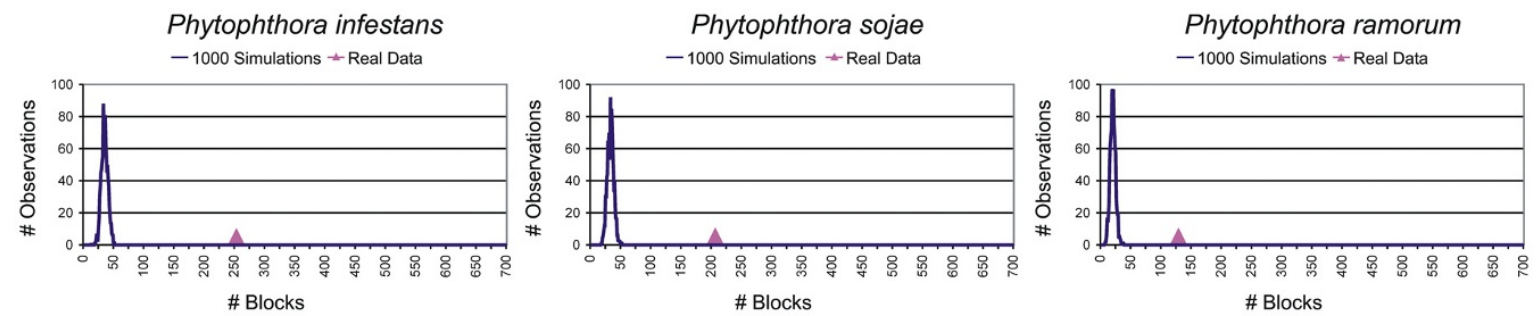

B Number of blocks with three homologous gene pairs, without intervening genes (3HOM)
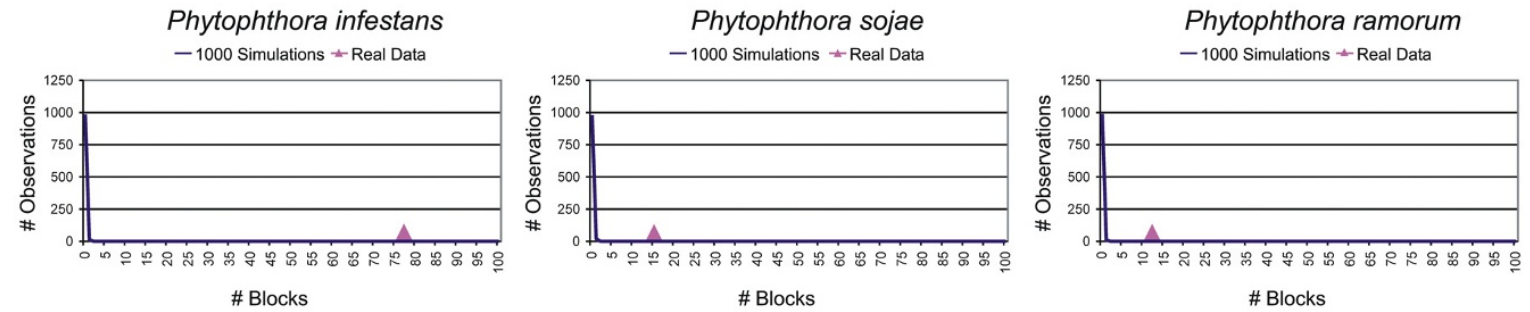

C Conservation of orientation in the duplicated blocks with two homologous gene pairs (2HOM)
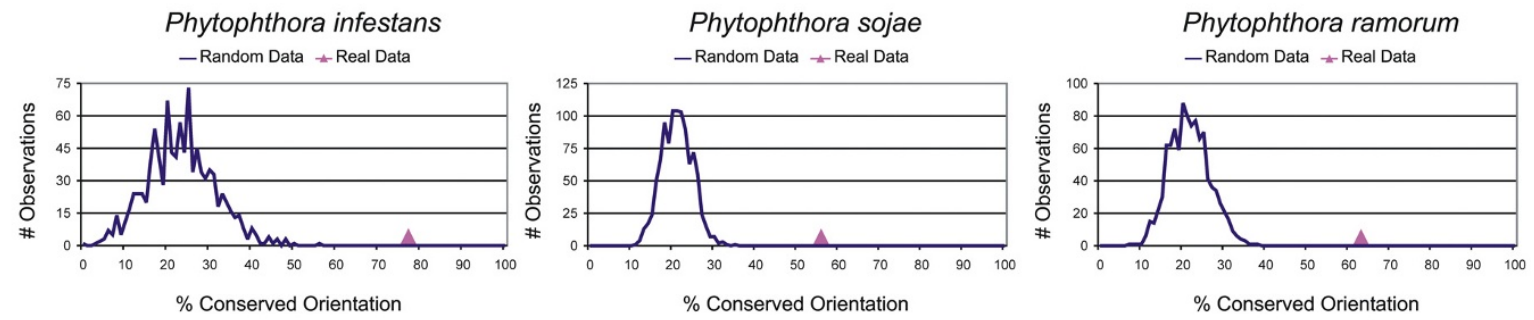

Figure 2 Duplicated blocks and orientation analysis in Phytophthora and random genomes. The number of (A) $2 \mathrm{HOM}$ blocks and (B) $3 \mathrm{HOM}$ blocks in P. infestans, P. sojae, and P. ramorum (pink triangles) versus the number of $2 \mathrm{HOM}$, resp. $3 \mathrm{HOM}$ blocks in random genomes, i.e. the expected number of blocks based on 1000 simulations (blue distribution). (C) Orientation conservation in the $2 \mathrm{HOM}$ and $3 \mathrm{HOM}$ blocks in real versus random data.

that most gene duplicates have been created at about the same time, this provides additional evidence for a single duplication event [23]. Therefore, we have tried to date the Phytophthora paranomes based on third codon or synonymous substitution rates (or Ks-estimation, see Methods). Because most substitutions in third-codon positions do not result in amino-acid replacements, the rate of fixation of these substitutions is expected to be relatively constant in different protein-coding genes [41] and, therefore, to reflect the overall mutation rate [42].

As can be seen in Figure 4 (grey line), the most recent speciation event is the divergence between $P$. ramorum and $P$. sojae. Prior to this event, $P$. infestans diverged from the common ancestor of $P$. sojae and P. ramorum (grey dotted line), which is in accordance with phylogenetic data [43]. Because of the fact that we only find small duplicated blocks, possibly because of many genome rearrangements (see further), many paralogs that arose through this large-scale duplication event will no longer lie in duplicated blocks. Therefore, we not only dated the homologous gene pairs still residing in duplicated blocks (Figure 4, dark blue shading), but also dated the whole paranome of the three species (Figure 4, light blue shading). As shown in Figure 4, both distributions have the same shape (it should be noted that the absolute values of the 'paranome' distribution are of course much higher). It is clear that in all three species there is a peak around $\mathrm{K}_{\mathrm{S}^{-}}$ values of 1.5-2.0. Although we are aware that higher values of $\mathrm{K}_{\mathrm{S}}$ should be interpreted with caution due to saturation effects, it is clear that many paralogs arose around the same time in the three species and prior to two speciation events, and must thus have occurred in their com- 


\begin{tabular}{|c|c|c|c|c|}
\hline$-P$ infestans & $-P$ sojae & - - P. ramorum & $-P$. tricornutum & $\neg-P$. falciparum \\
\hline- S. cerevisiae & - -K. lactis & - A. thaliana & - - H. sapiens & $\neg-T$. nigroviridis \\
\hline- - C. elegans & $\rightarrow$ D. melan & - A. gambiae & & \\
\hline
\end{tabular}

A Number of blocks with two homologous gene pairs (2HOM), without intervening genes

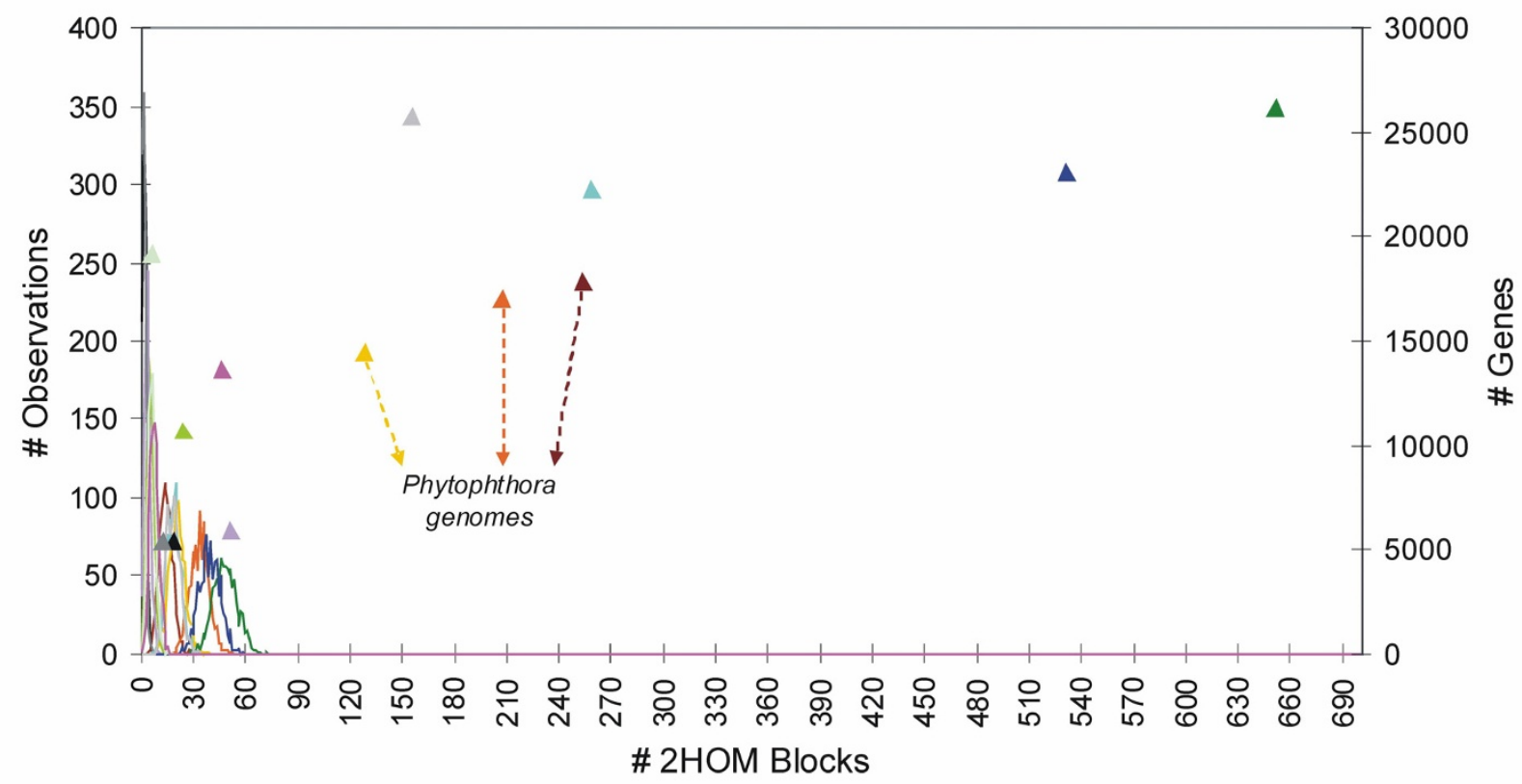

B Percentage of blocks with two homologous gene pairs (2HOM), without intervening genes

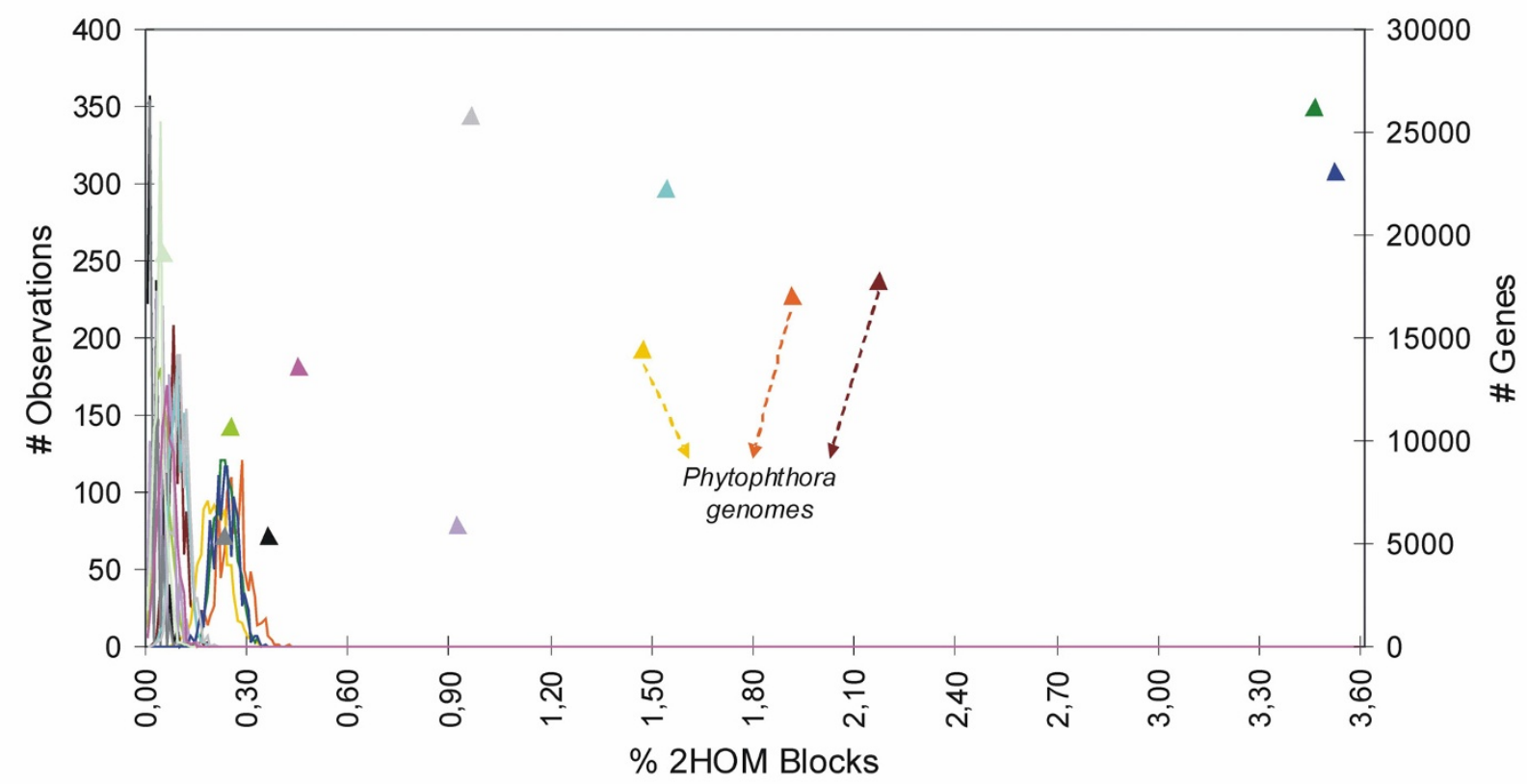

Figure 3 Number (A) and percentage (B) of 2HOM blocks in the Phytophthora species and reference genomes. The results for real genomes are represented by triangles, the results for the corresponding randomized genomes are represented by the distributions in the same color as the triangle data points. The left $Y$-axis applies to the random data, the right $Y$-axis applies to the real data. 


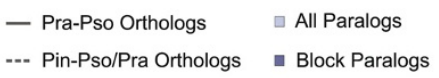

A Phytophthora infestans

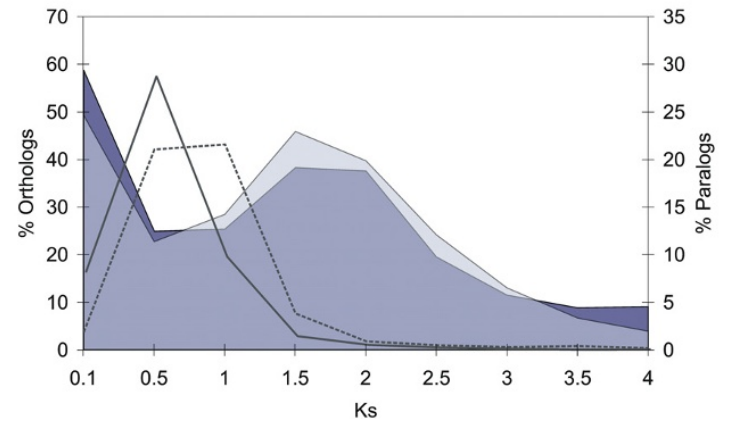

B Phytophthora sojae

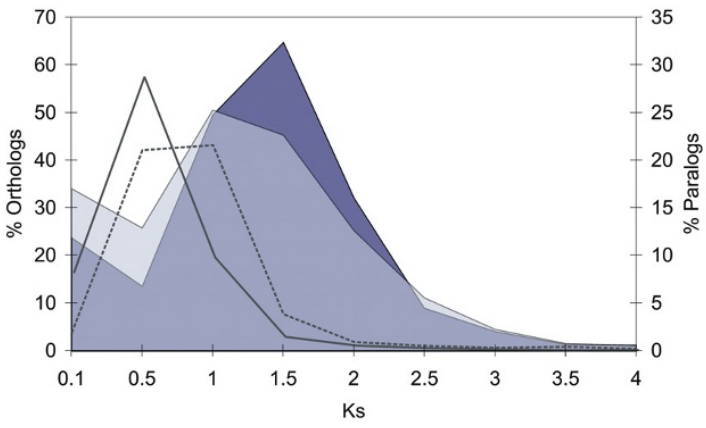

C Phytophthora ramorum

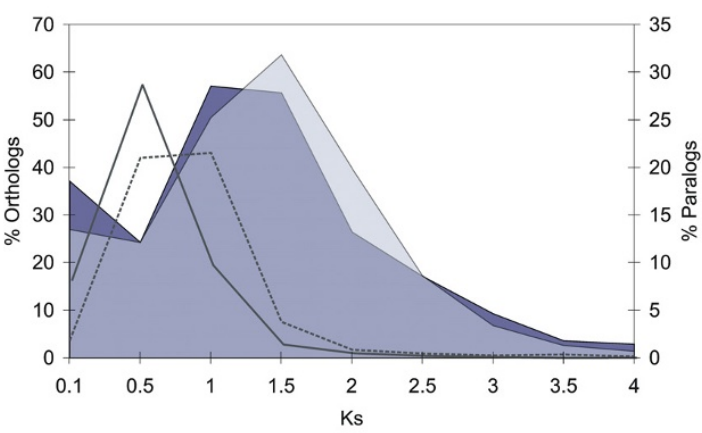

Figure $4 \mathrm{~K}_{\mathrm{S}}$ dating of the Phytophthora duplication and speciation events. $K_{5}$ distribution for paralogous and orthologous genes in the (A) P. infestans, (B) P. sojae and (C) P. ramorum genome. The $\mathrm{K}_{\mathrm{S}}$ distribution for the P. sojae (Pso) and P. ramorum (Pra) orthologs (left Y-axis), representing the $P$. sojae-P. ramorum speciation, is indicated by a full grey line. The $K_{S}$ distribution for the $P$. sojae (or $P$. ramorum) and $P$. infestans (Pin) orthologs (left $Y$-axis), representing the $P$. infestans-P. sojae/P. ramorum speciation, is indicated by a grey dotted line. The $\mathrm{K}_{5}$ distribution of the whole paranome (right $Y$-axis) is shown in light blue shading; the $\mathrm{K}_{\mathrm{S}}$ distribution of the paralogs located in duplicated blocks (right $\mathrm{Y}$-axis) is indicated in dark blue shading.

mon ancestor. This is also confirmed by the fact that $P$. sojae and $P$. ramorum still share $242 \mathrm{HOM}$ blocks (i.e., $11.6 \%$ resp. $18.8 \%$ of the total number of $P$. sojae resp. $P$. ramorum $2 \mathrm{HOM}$ blocks). $P$. sojae and $P$. ramorum also share 19 (9.2\%) resp. 11 (or 8.6\%) of their 2HOM blocks with $P$. infestans.

\section{Gene orientation conservation in the duplicated blocks}

We also studied gene orientation conservation in the Phytophthora and reference genomes. To this end, we have applied the strategy shown in Figure 1 (Panel C and D). In Panel $C$, the possible situations are shown where the orientation of the genes in 2HOM blocks is completely conserved. Since it is possible that during a WGD whole regions are being inverted, the different possibilities shown in Panel D are also considered conserved. All the other cases, where only one gene is inverted and the other one not etc., we define as the orientation not to be conserved. We have applied this strategy to all the genomes in our dataset and again ran 1000 simulations for every species. As can be seen in Figure 2C, $77 \%$ of the $2 \mathrm{HOM}$ blocks in $P$. infestans have a conserved gene orientation (pink triangle), whereas the conserved orientation in the reshuffled genomes is much smaller (blue line). In P. sojae and $P$. ramorum the conservation percentage is slightly lower, but still higher than in random data. In A. thaliana, T. nigroviridis and H. sapiens, again the situation is similar as in Phytophthora, although in human and Tetraodon the percentages are a bit higher (see Additional file 7). In C. elegans and D. melanogaster the conservation percentages are just below 50 and at the tail of the simulation curve. Also in A. gambiae the conservation percentage is at the tail of the random curve. For the other genomes it is difficult to conclude anything because there are too few data points. This is also the reason why this simulation analysis was not performed on the $3 \mathrm{HOM}$ blocks.

The fact that the blocks in the Phytophthora species have a conserved orientation provides further support for the homologous gene pairs to have been duplicated in concert. If the homologous gene pairs would have been duplicated separately and afterwards assembled into gene clusters for example, then the genes within the block could have easily been inverted, resulting in a non-conserved gene orientation within the block. Also Cavalcanti and colleagues showed that in yeast the number of blocks with the same gene order was similar to the number of blocks with the same gene order and gene orientation, while in C. elegans the number of blocks dropped substantially after imposing the orientation criterion [44].

\section{Gene retention following duplication in Phytophthora}

Besides block duplicates, also the number of tandem duplicates is large in the Phytophthora genomes. In pathogenic species, it is well-known that genes related to pathogenesis are often located in tandem arrays (for example [19,45-47]. In a previous study, this was for example observed for $P$. falciparum, but also for both $P$. sojae and $P$. ramorum [19]. To see whether there is a simi- 
lar bias in gene function for genes retained in the 2- and 3HOM blocks, we calculated GO Slim enrichments (see Methods) for both the tandem and block duplicate datasets. Table 2 shows the GO Slim labels that are significantly $(Q$-value $<0.05)$ enriched in (i) tandem duplicates, (ii) block duplicates, and (iii) in both tandem and duplicates.

As expected, and previously shown [19], tandem duplicates are enriched in genes related to pathogenesis, such as genes involved in symbiosis and genes with specific kinase activity. Moreover, $25 \%$ of all genes annotated with the GO-term "symbiosis" are part of tandem gene clusters (see Table 2). When we consider the GO-tree Cellular Component $(\mathrm{C})$, we observe that tandem genes are often expressed in lysosomes, vacuoles, the external encapsulating structures, the cell wall and the extracellular region, which refers to the outermost structure of a cell (or the host cell environment in the case of an intracellular parasite).

The block duplicates are specifically enriched in the processes cell communication and signal transduction and in the functions actin binding and calcium ion binding, signal transducer activity, transcription regulator activity and receptor activity. Many of these functions, such as signal transduction and transcription but also calcium binding have been shown in several studies to be preferentially retained after a whole genome duplication because of gene dosage and gene balance effects [48-53]. Therefore, the specific retention of these genes in the small duplicated blocks in Phytophthora provides additional evidence for a WGD, rather than individual segmental duplications, where we would expect the opposite [51]. Additionally, the retention of calcium binding, signal transduction and cell communication proteins may also have been important in the infection process of the plant pathogen. It has been shown that the plant pathogen Phytophthora parasitica forms, at the site of infection, biofilms that contribute to disease development [54]. These biofilms protect the pathogen against plant defence responses and fungicidal treatments and use cell-cell communication to promote the exchange of signals and nutrients between, among others, sessile and planktonic zoospores [54]. Calcium, for example, is one of the candidate substances responsible for the chemotaxis of zoospores toward previously encysted zoospores [55-57]. Furthermore, the encystment of zoospores and the germination of cysts to form hyphae is also stimulated by nutrients and calcium (reviewed in [58]). Regarding the GO-tree cellular component, we see no preference of expression in the extracellular regions.

It is clear that both tandem and block duplicates are enriched in genes that play a role in pathogenesis. Additionally, both types of duplicates are enriched in genes that are important in the response to external, biotic and abiotic stimulus and stress. Also genes with hydrolase, transporter and catalytic activity, of which many are linked to pathogenesis, are enriched in both categories of duplicates. For example, genes of the glycosyl hydrolase family encode extracellular enzymes capable of hydrolyzing the xyloglucan component of the host cell wall, thereby facilitating the pathogen physical penetration process [59]. Although the large majority of these wellknown pathogenicity genes [12,13] have clearly evolved through a continuous process of tandem duplications, we have now identified some of them as remainders of an older large-scale duplication event.

\section{Discussion}

All three Phytophthora genomes contain many more small duplicated blocks than would be expected by chance alone. Furthermore, when we compare the number of duplicated blocks with those of organisms that have most probably not undergone large scale duplication events (e.g. Drosophila melanogaster or Phaeodactylum tricornutum), the difference is obvious (see Figure 3 and Table 1). Moreover, we also observed a clear difference with organisms that did undergo some segmental duplications, but no WGD. For example, Plasmodium falciparum, the causative agent of severe human malaria, carries multiple segmental duplications in the otherwise highly variable subtelomeres of its chromosomes [37]. However, the number of $2 \mathrm{HOM}$ and $3 \mathrm{HOM}$ blocks detected is still much smaller than in Phytophthora. Also in $K$. lactis, a yeast species that has not undergone a WGD, but for which eight segmental duplications have been documented, on top of some segmental duplications at the subtelomeres [39], the number of detected small duplicated blocks is much less than in Phytophthora [38]. On the other hand, the number of $2 \mathrm{HOM}$ blocks in $C$. elegans, which has undergone a few segmental duplications [36], is higher than in P. ramorum, but still considerably lower than in $P$. infestans and $P$. sojae. The number of 3HOM blocks on the other hand is higher than in $P$. ramorum and $P$. sojae but still lower than in $P$. infestans. However, it should be noted that the large number of $2 \mathrm{HOM}$ and $3 \mathrm{HOM}$ blocks in C. elegans is mainly due to a few larger segmental duplications involving between 10 and 26 genes [36]. It is also important to note that the duplicated blocks in all three Phytophthora species are spread over more than 60 percent of the number of scaffolds and we did not observe a bias to certain scaffolds, only a correlation between the size of the scaffold and the number of duplicated blocks, something we would expect for a WGD event. On the other hand, in C. elegans, 70\% of the segmental duplications are intrachromosomal [36].

Because the number of blocks is directly correlated with, among other things, (i) the number of genes, (ii) the extent of genome rearrangements, and (iii) the quality of 
Table 2: Significantly enriched GO-labels in the block and/or tandem duplicates of the Phytophthora species.

\begin{tabular}{|c|c|c|c|c|c|c|c|c|}
\hline \multirow[t]{2}{*}{ GO-tree } & \multirow[t]{2}{*}{ GO-label } & \multirow[t]{2}{*}{ GO-description } & \multirow[t]{2}{*}{ No. of TD* } & \multirow[t]{2}{*}{ No. of BD* } & \multicolumn{2}{|c|}{$\begin{array}{l}\text { Relative to } \\
\text { the total No. } \\
\text { of TD and BD }\end{array}$} & \multicolumn{2}{|c|}{$\begin{array}{c}\text { Relative to } \\
\text { the total No. } \\
\text { of genes }\end{array}$} \\
\hline & & & & & $\%$ TD & $\%$ BD & $\%$ TD & $\%$ BD \\
\hline \multicolumn{9}{|c|}{ Only in tandem duplicates } \\
\hline$P$ & GO:0006464 & protein modification process & 173 & 18 & 14.9 & 5.4 & 9.4 & 1.0 \\
\hline$P$ & GO:0044403 & $\begin{array}{l}\text { symbiosis, encompassing mutualism through } \\
\text { parasitism }\end{array}$ & 28 & 4 & 2.4 & 1.2 & 25.0 & 3.6 \\
\hline$P$ & GO:0006091 & generation of precursor metabolites and energy & 97 & 22 & 8.4 & 6.6 & 10.8 & 2.4 \\
\hline $\mathrm{F}$ & GO:0016301 & kinase activity & 163 & 15 & 10.4 & 3.7 & 10.5 & 1.0 \\
\hline $\mathrm{F}$ & GO:0008289 & lipid binding & 49 & 7 & 3.1 & 1.7 & 8.9 & 1.3 \\
\hline $\mathrm{F}$ & GO:0004672 & protein kinase activity & 155 & 15 & 9.9 & 3.7 & 12.2 & 1.2 \\
\hline C & GO:0005576 & extracellular region & 56 & 10 & 10.2 & 5.2 & 21.5 & 0.4 \\
\hline C & GO:0005764 & lysosome & 12 & 3 & 2.2 & 1.5 & 37.5 & 9.4 \\
\hline C & GO:0005773 & vacuole & 12 & 3 & 2.2 & 1.5 & 31.6 & 7.9 \\
\hline C & GO:0030312 & external encapsulating structure & 8 & 3 & 1.5 & 1.5 & 20.5 & 7.7 \\
\hline C & GO:0005618 & cell wall & 7 & 3 & 1.3 & 1.5 & 20 & 8.6 \\
\hline
\end{tabular}

\section{Only in block duplicates}

\begin{tabular}{|c|c|c|c|c|c|c|c|c|}
\hline$P$ & GO:0006996 & organelle organization and biogenesis & 45 & 26 & 3.9 & 7.8 & 5.8 & 3.3 \\
\hline$P$ & GO:0007154 & cell communication & 53 & 24 & 4.6 & 7.2 & 5.9 & 2.7 \\
\hline$P$ & GO:0007165 & signal transduction & 19 & 22 & 1.6 & 6.6 & 3.2 & 3.7 \\
\hline$P$ & GO:0015031 & protein transport & 4 & 12 & 0.3 & 3.6 & 1.0 & 3.0 \\
\hline$P$ & GO:0016043 & cellular component organization and biogenesis & 59 & 45 & 5.1 & 13.5 & 4.5 & 3.4 \\
\hline $\mathrm{F}$ & GO:0003779 & actin binding & 8 & 8 & 0.5 & 2.0 & 9.1 & 9.1 \\
\hline $\mathrm{F}$ & GO:0004871 & signal transducer activity & 10 & 15 & 0.6 & 3.7 & 4.0 & 5.9 \\
\hline $\mathrm{F}$ & GO:0004872 & receptor activity & 10 & 14 & 0.6 & 3.5 & 7.4 & 10.3 \\
\hline $\mathrm{F}$ & GO:0005509 & calcium ion binding & 13 & 19 & 0.8 & 4.7 & 2.1 & 3.0 \\
\hline
\end{tabular}


Table 2: Significantly enriched GO-labels in the block and/or tandem duplicates of the Phytophthora species. (Continued)

\begin{tabular}{|c|c|c|c|c|c|c|c|c|}
\hline $\mathrm{F}$ & GO:0008092 & cytoskeletal protein binding & 8 & 8 & 0.5 & 2.0 & 8.1 & 8.1 \\
\hline $\mathrm{F}$ & GO:0030528 & transcription regulator activity & 29 & 12 & 1.9 & 3.0 & 7.0 & 2.9 \\
\hline \multicolumn{9}{|c|}{ In tandem and block duplicates } \\
\hline$P$ & GO:0005975 & carbohydrate metabolic process & 174 & 39 & 15.0 & 11.7 & 19.2 & 4.3 \\
\hline$P$ & GO:0006810 & transport & 326 & 120 & 28.1 & 35.9 & 10.5 & 3.9 \\
\hline$P$ & GO:0006811 & ion transport & 53 & 29 & 4.6 & 8.7 & 9.0 & 4.9 \\
\hline$P$ & GO:0006950 & response to stress & 53 & 20 & 4.6 & 6.0 & 12.0 & 4.5 \\
\hline$P$ & GO:0009605 & response to external stimulus & 10 & 8 & 0.9 & 2.3 & 20.0 & 16.0 \\
\hline$P$ & GO:0009628 & response to abiotic stimulus & 20 & 9 & 1.7 & 2.6 & 16.1 & 7.3 \\
\hline$P$ & GO:0009607 & response to biotic stimulus & 36 & 8 & 3.1 & 2.3 & 23.1 & 5.1 \\
\hline $\mathrm{F}$ & GO:0003824 & catalytic activity & 1019 & 253 & 65.2 & 62.6 & 8.8 & 2.2 \\
\hline $\mathrm{F}$ & GO:0005215 & transporter activity & 215 & 74 & 13.8 & 18.3 & 13.0 & 4.5 \\
\hline $\mathrm{F}$ & GO:0016787 & hydrolase activity & 446 & 125 & 28.6 & 31.0 & 9.5 & 2.7 \\
\hline
\end{tabular}

Both P-value and Q-value for the GO-labels shown are lower than 0.05 .

${ }^{*} \mathrm{TD}=$ Tandem Duplicates; BD = Block Duplicates

the genome assembly, we have to take these issues into account. For example, as stated before, the number of blocks in the paleopolyploid S. cerevisiae was lower than expected. However, this is explained by the fact that, compared to the other genomes used in this study, yeast has much fewer genes. On top of that, S. cerevisiae has undergone many rearrangements $[28,38,60]$. Figure $3 B$ shows the percentage of $2 \mathrm{HOM}$ blocks for the different genomes analyzed, taking into account the number of blocks that theoretically can be found if the whole genome would have been duplicated and there would have been no genome rearrangements (translocation, loss, ...). In practice, if a complete chromosome (or scaffold in our case) with $x$ genes has been duplicated, we would expect to find $(x-1) 2 \mathrm{HOM}$ and $(x-2) 3 \mathrm{HOM}$ blocks, provided none of the duplicated genes would have been translocated or lost nor other genes inserted. By dividing the number of identified blocks by the number of possible blocks, we obtain the relative number of duplicated blocks for all genomes (see Figure 3B and Table 1). Regarding 2HOM blocks, all genomes that have not undergone a large-scale duplication event, have values below $0.5 \%$. The same is true for Plasmodium falciparum and Kluyveromyces lactis, which have only undergone some segmental duplications. For the other species, except $S$. cerevisiae, which are known to have undergone at least one genome duplication, the percentages are all $>1.5 \%$, including $P$. infestans $(2.17 \%)$ and $P$. sojae $(1.91 \%)$. P. ramorum is just $<1.5 \%$ (1.47\%), but there are no non-duplicated or segmentally duplicated genomes with a value larger than $1 \%$. It should also be noted that, when taking the number of genes into account, the difference in the number of $2 \mathrm{HOM}$ blocks between S. cerevisiae and the non-duplicated organisms becomes larger. Also the percentage of blocks in all three Phytophthora species is now greater than in C. elegans. Moreover, the relative number of blocks in H. sapiens is smaller than in $P$. infestans and $P$. sojae, and similar to $P$. ramorum. Both Tetraodon and Arabidopsis still have the highest relative number of blocks.

For the 3HOM blocks, the difference between organisms that have undergone large-scale duplications and 
those that have not is even more pronounced (also see Table 1). For all non-duplicated genomes, the percentages are below $0.05 \%$. When we consider the other genomes, the relative number of $3 \mathrm{HOM}$ blocks in $P$. infestans is the highest. For P. sojae and P. ramorum however, the percentages are lower than for Arabidopsis and Tetraodon, similar to S. cerevisiae, H. sapiens and C. elegans, and higher than for the other reference organisms.

It is important to realize that the genomes of all three Phytophthora's still consist of scaffolds, whereas most other genomes discussed here have already been assembled into chromosomes. If the majority of scaffolds are rather small, it is obvious that it is much harder to detect large duplicated regions than for genomes that are well assembled (e.g. Arabidopsis, human, Tetraodon, Drosophila, ...). Therefore, we considered the distribution of scaffold sizes (i.e. number of genes on an annotated scaffold) in the different Phytophthora species and compared them with the diatom Phaeodactylum tricornutum, a genome that is also not yet assembled into chromosomes (see Figure 5). For every size bin, we counted the percentage of genes of the corresponding genome located on a scaffold of that size. In P. ramorum (blue histogram), we observed that the percentage of genes lying on a small scaffold (i.e. between 1 and 25 genes) is greater than for the other genomes. Also when we consider the cumulative percentage (blue line), we see that all genes (i.e. $100 \%$ ) are found on scaffolds with a size smaller than 400 genes, whereas in Phaeodactylum we have scaffolds with more than 900 genes and in $P$. infestans with more than 1000 genes. The situation in P. sojae is similar to that of $P$. ramorum, although slightly better. In Phaeodactylum, the number of genes on small scaffolds is the lowest. Therefore because in Phaeodactylum the average scaffold sizes are larger, it should be easier to detect larger duplicated blocks. The fact that we could hardly find any duplicated blocks in Phaeodactylum tricornutum (Table 1 and Additional file 8), whereas we do find many in P. infestans and a considerable number in $P$. ramorum and $P$. sojae again provides support for Phytophthora species having undergone a large-scale or even entire genome duplication event. In addition, since the number of blocks found in the three species is linearly correlated with the assembly quality, it is likely that in P. ramorum and $P$. sojae the duplication signal would be more similar to $P$. infestans after improving the genome assembly.

\section{Conclusion}

Analyses of the Phytophthora genomes seem to suggest that these organisms have undergone a large-scale gene duplication or WGD in their evolutionary past. Likely, this event has been shared by all three Phytophthora species, $P$. infestans, $P$. ramorum, and $P$. sojae and thus occurred before their speciation. Although we cannot exclude that the many small duplicated blocks have been created through many independent small block duplications, we do consider this less likely. First, when we calculate the age of the duplicated blocks a large fraction seems to have originated at the same time and they seem to be very old. If the many small blocks observed in the different Phytophthora genomes would have been created by a continuous mode of segmental duplications, we would expect to see an exponential decrease when plotting the age of the duplicated blocks against their frequency (i.e., many young blocks, few old ones), which is not what we observe $[23,51]$. It could still be that a majority of segmental duplications occurred in a short time interval in the common ancestor of all three Phytophthora species, but this scenario is certainly much less parsimonious than a single WGD. Furthermore, the specific enrichment of regulatory genes in the duplicated blocks provides additional support for a WGD, rather than many smaller segmental duplication events, after which where we would expect strong selection against retention of such genes [50-53,61-64].

Second, polyploids have already been identified within several species of Phytophthora [65-69] and other oomycetes [70] providing additional support that $P$. infestans could indeed be an ancient polyploid (with a now diploidized genome). The findings of Sansome (1977) suggested that $P$. infestans may exist in nature in the tetraploid condition and that this tetraploid might be better adapted, for instance to cooler conditions [65]. The author also claimed that the discovery of many pathogenic races of $P$. infestans [71] may be related to polyploidy in $P$. infestans [65]. The fact that we also find many genes related to pathogenesis in our set of retained duplicates might actually confirm this hypothesis.

Therefore, we conclude that Phytophthora is most likely an ancient polyploid. The fact that many small blocks are found suggests that its genome has been heavily rearranged following the duplication event. Furthermore, the observation that the Phytophthora genomes have a high repeat content, and that the gene order conservation between the genomes drops when the repeat content increases [13], further suggests that those repeats have played an important role in the rearrangement process. Haas and colleagues (2009) also suggested that the high rate of transposon activity must have occurred more recently [13], supporting our hypothesis that the WGD event has preceded the rearrangement processes. As a consequence, after tens of millions of years of evolution, and in particular for fast evolving genomes of pathogens, the paucity of a considerable number of retained homologous gene pairs in close proximity makes it almost impossible to detect statistically significant collinear regions. This might explain why no evidence has been found previously for WGD or large-scale segmental duplications in 


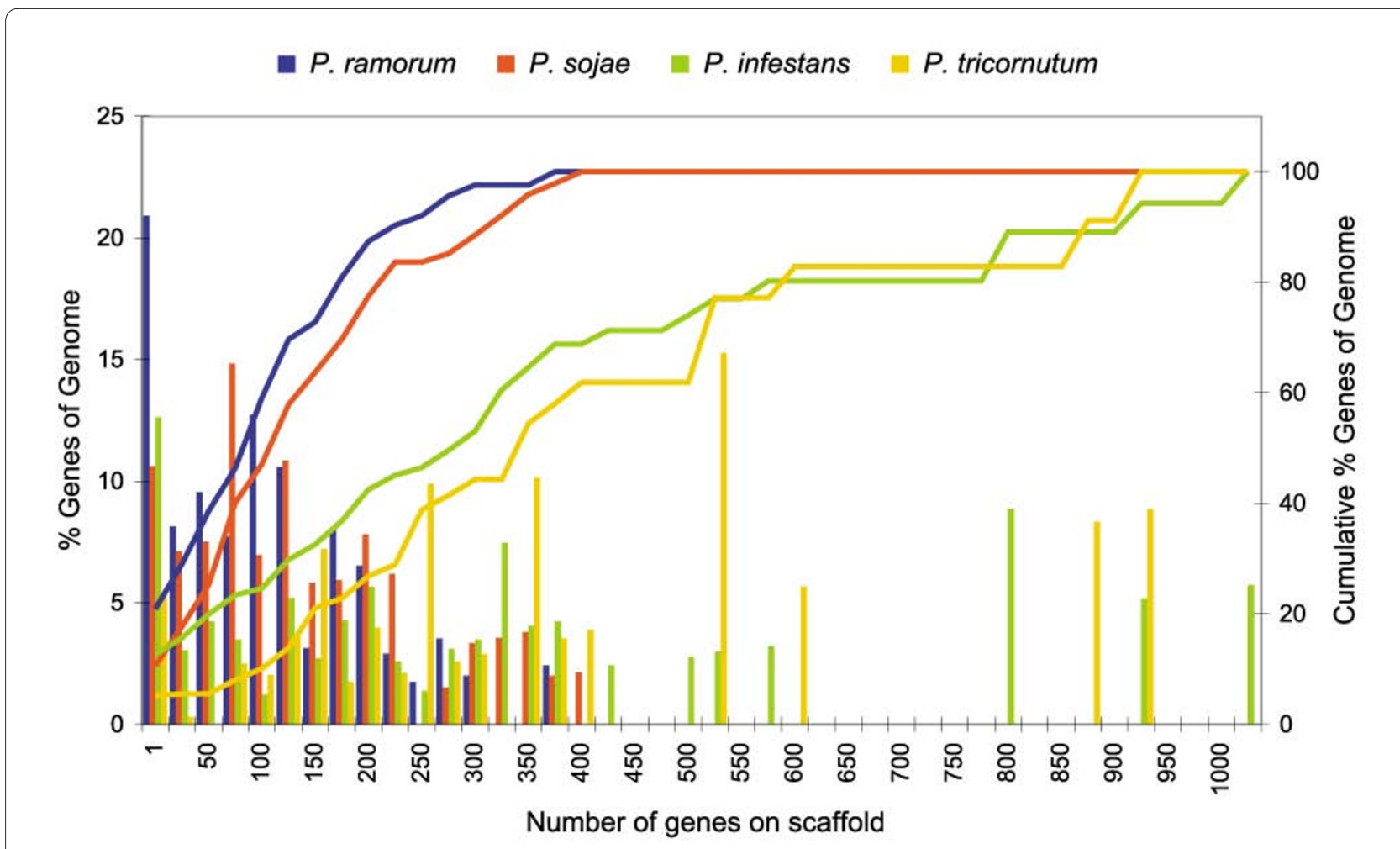

Figure 5 Quality of the genome assembly for the Phytophthora species and for the diatom Phaeodactylum tricornutum. The histograms represent the percentage of genes (left Y-axis) lying on a scaffold of a certain size, measured by the number of genes on a scaffold. The corresponding lines represent the cumulative percentage of genes (right $Y$-axis).

the Phytophthora species [12,13]. However, our newly developed strategy to look for large numbers of small duplicated blocks and compare these with genomes of other organisms for which the duplication past is better known, might still unveil ancient polyploidy events.

\section{Methods}

\section{Construction of the datasets and defining homologs}

The predicted protein sequences of three Phytophthora species, namely Phytophthora sojae (JGI, v1.1), Phytophthora ramorum (JGI, v1.1) and Phytophthora infestans (v1, http://www.broad.mit.edu/annotation/genome/ phytophthora infestans/) were downloaded, as well as the predicted protein sets of Phaeodactylum tricornutum (JGI, v1.0), Plasmodium falciparum (Plasmodb), Arabidopsis thaliana (TIGR, Release 5), Kluyveromyces lactis (NCBI), Saccharomyces cerevisiae http://www.yeastgenome.org, Anopheles gambiae (Ensembl, Release 52), Caenorhabditis elegans (Ensembl, Release 31.140), Drosophila melanogaster (Ensembl, Release 31.3e), Homo sapiens (Ensembl, Release 35) and Tetraodon nigroviridis (Ensembl, Release 53).

If alternative splice variants were detected for one gene, only the longest transcript was used. Also transposon-like genes were removed based on homology with known trans- posons retrieved from the EMBL Nucleotide Sequence Bank http://www.ebi.ac.uk/embl/ and the Swiss-Prot Protein KnowledgeBase http://www.expasy.ch/sprot/. To identify homologous genes, a similarity search was performed for every genome (BLASTP [72]; E-value cutoff E-10). Next, gene families were built with MCLBLASTLINE (Inflation Factor of 2.0; http://micans.org/mcl/, $[73,74]$.

\section{Dating of paralogous and orthologous gene pairs}

The fraction of synonymous substitutions per synonymous site $\left(K_{S}\right)$ is used to estimate the time of duplication or speciation between two paralogous resp. orthologous sequences. All pairwise alignments of the paralogous or orthologous nucleotide sequences belonging to a gene family were made by using CLUSTALW [75]. Gaps and adjacent divergent positions in the alignments were removed. $K_{S}$ estimates were obtained with the CODEML program [76] of the PAML package [77]. Calculations were repeated ten times to avoid incorrect $\mathrm{K}_{\mathrm{S}}$ estimations because of suboptimal local maxima. To exclude gene pairs that can be the result of redundancy instead of duplication, only gene pairs with a $\mathrm{K}_{\mathrm{S}}$ estimate higher than 0.1 were considered for further evaluation. 


\section{Detection of (large) duplicated regions ( $>2$ genes)}

Duplicated regions in the Phytophthora and reference genomes were identified with the i-ADHoRe software [24]. Homologous gene pairs, defined by MCLBLASTLINE, served as an input for the i-ADHoRe algorithm. Gene pairs of gene families with more than 100 members were omitted from the analysis. The following parameters were used: gap size of 10 genes; cluster gap of 20 genes; P-value of 0,001; Q-value of 0.9 and a minimum of three homologs to define a duplicated block.

\section{Detection of tandem genes and small duplicated blocks $<$ 4 genes)}

Based on the MCLBLASTLINE-output, the order of proteins on a scaffold was converted into an order of gene families A, B, C, ..., while keeping track of the original protein IDs (see Figure 1, Panel A). Scaffolds with fewer than 6 genes were omitted from further analyses. To define all existing gene family pairs that occur next to each other in the genome, a window size of two was used to scan every scaffold. Tandem gene family pairs were excluded. Thus, in a string of, for example, A-B-C-C-B-A we define $A B$ (BA is remapped to $A B)$ and $B C(C B$ is remapped to $B C$ ) as gene family pairs. $C C$ is a tandempair so this pair was discarded for the block analysis and analyzed separately. With the gene pairs identified this way, we again scan every scaffold to count how many times this gene pair was found. The search is also done with a window size of 1 but when a pair is found, we move with a window size of two for the next search step only, to prevent that we would count $A B$ two times in the example $A B A C D$ (remember that $B A$ is remapped to $A B$ ). Therefore, when we detect $A B$, we jump one window further to take AC as the next pair instead of BA. When a pair is found more than once, we call it a block with two homologs (or 2HOM block). Finally, for all gene pairs that are detected more than once, a unique block ID is defined. In a post-processing step, duplicated blocks where at least one of the homologous gene pairs is a member of a large gene family ( $>100$ genes) were omitted from the analysis. Also duplicated blocks where one of the gene pairs has a $\mathrm{K}_{\mathrm{S}}$ estimate lower than 0.1 were removed to reduce the effect of redundancy. The gene IDs and coordinates of the gene pairs located in $2 \mathrm{HOM}$ blocks can be found in Additional file 8 .

A similar strategy was applied to detect blocks with three homologous genes or 3HOM Blocks (Figure 1, Panel B). So ABC, ABB...BBBC and CBA are all remapped to $A B C$. However, $B A C$ is not remapped to $A B C$. Also note that $2 \mathrm{HOM}$ blocks mean that there must be at least two successive homologs, so in the set of 3HOM blocks, the $2 \mathrm{HOM}$ blocks are also included. The gene IDs and coordinates of the gene pairs located in 3HOM blocks can be found in Additional file 9.

\section{Validation of small duplicated blocks}

To examine if the number of blocks that we observe is different from what we would expect by chance only, we ran 1000 simulations for every genome. In brief, in every genome the tandem duplicates were remapped to the first gene and the gene families with more than 100 genes were removed. Next, every genome was shuffled 1000 times and each time the number of detected $2 \mathrm{HOM}$ and $3 \mathrm{HOM}$ blocks was counted. If the number of detected blocks is greater in the real data than in random data, we can conclude that the number of blocks found is significantly higher than we could expect by chance only.

\section{Conservation of gene orientation}

For all 2HOM blocks, we compared the order of gene orientation between both homologous segments. If the gene orientation and gene order were conserved between both homologous segments (see Figure 1, Panel C) then we concluded that the orientation is conserved. If the gene order is inverted together with the orientation (see Figure 1 , Panel D), then we also conclude that the orientation in this block is conserved. In all other cases, we consider the orientation as not conserved. The same analysis was done on all randomized (shuffled) genomes created for the block detection strategy.

\section{GO annotation and GO enrichment}

The proteins of all Phytophthora genomes were annotated using Gene Ontology (GO) [78]. In a first step, all genes were annotated for protein function using InterProScan [79]. Next, the resulting InterPro annotation was converted into GO annotation. Proteins mapped to a particular GO category were also explicitly included into all parental categories. All GO categories were also mapped into the GO Slim categories. The statistical significance of functional GO Slim enrichment was evaluated by using the hypergeometric distribution, whereas multiple hypotheses testing was done by using FDR [80].

\section{Additional material}

Additional file 1 Detection of 2HOM blocks in the Phytophthora and reference genomes. The real data are represented by the pink triangle data points, while the 1000 simulations or random data is represented by blue curves. The number of observations in the real data is always equal to 1 since there is only one real dataset per genome, while there are 1000 random datasets per genome.

Additional file 2 Detection of $3 \mathrm{HOM}$ blocks in the Phytophthora and reference genomes. Interpretation is as in Additional file 1.

Additional file 3 Frequency of scaffolds with a certain number of duplicated blocks.

Additional file 4 Relation between the size of a scaffold and the number of detected blocks. The graphs with the yellow bars show the relation between the number of detected $2 \mathrm{HOM}$ blocks and the average scaffold size for all three Phytophthora species.

Additional file $\mathbf{5}$ Supporting methods and results. This file contains additional information about the applied methods and the results regarding the functional clustering analysis. 


\section{Additional file 6 Intergenic distances in the duplicated blocks. Com- parison of the Intergenic Distances in $2 \mathrm{HOM}$ Blocks (pink), $3 \mathrm{HOM}$ Blocks (green) and the whole proteome (blue) in the Phytophthora and reference genomes. \\ Additional file 7 Orientation conservation in the $2 \mathrm{HOM}$ and $3 \mathrm{HOM}$ blocks of the Phytophthora and reference genomes. Interpretation is as in Additional file 1. \\ Additional file 8 Gene IDs and gene coordinates of the Phytophthora duplicates located in $2 \mathrm{HOM}$ blocks. \\ Additional File 9 GeneIDs and gene coordinates of the Phytophthora duplicates located in $3 \mathrm{HOM}$ blocks}

\section{Authors' contributions}

CM designed the research, analyzed the data and wrote the paper. YVdP designed the research, supervised the project and wrote the paper.

\section{Acknowledgements}

The authors would like to thank Jonathan Gordon, Grigoris Amoutzias, Steven Maere and Klaas Vandepoele for helpful discussions. C.M. is indebted to the Institute for the Promotion of Innovation by Science and Technology in Flanders for a predoctoral fellowship. This work was supported by the Belgian Federal Science Policy Office: IUAP P6/25 (BioMaGNet). The funders had no role in study design, data collection and analysis, decision to publish, or preparation of the manuscript

\section{Author Details}

'Department of Plant Systems Biology, VIB, Technologiepark 927, B-9052 Ghent, Belgium and 2Bioinformatics and Evolutionary Genomics, Department of Molecular Genetics, Technologiepark 927, Ghent University, B-9052 Ghent, Belgium

Received: 4 February 2010 Accepted: 3 June 2010

Published: 3 June 2010

\section{References}

1. Govers F, Gijzen M: Phytophthora genomics: the plant destroyers' genome decoded. Molecular plant-microbe interactions 2006 19(12):1295-1301

2. Latijnhouwers $M$, de Wit PJ, Govers F: Oomycetes and fungi: similar weaponry to attack plants. Trends in microbiology 2003, 11(10):462-469.

3. Judelson HS, Blanco FA: The spores of Phytophthora: weapons of the plant destroyer. Nature reviews 2005, 3(1):47-58.

4. Baldauf SL, Roger AJ, Wenk-Siefert I, Doolittle WF: A kingdom-level phylogeny of eukaryotes based on combined protein data. Science (New York, NY) 2000, 290(5493):972-977.

5. Yoon HS, Hackett JD, Pinto G, Bhattacharya D: The single, ancient origin of chromist plastids. Proc Natl Acad Sci USA 2002, 99(24):15507-15512.

6. Cavalier-Smith T: Principles of protein and lipid targeting in secondary symbiogenesis: euglenoid, dinoflagellate, and sporozoan plastid origins and the eukaryote family tree. J Eukaryot Microbiol 1999, 46(4):347-366.

7. Keeling PJ: Diversity and evolutionary history of plastids and their hosts. Am J Bot 2004, 91:1481-1493.

8. Fry WE, Goodwin SB: Re-emergence of potato and tomato late blight in the United States. Plant Dis 1997, 81:1349-1357.

9. Ross D: Ireland: History of a Nation. New Lanark: Geddes \& Grosset Ltd 2002.

10. Grada CO: Ireland's Great Famine: Interdisciplinary Perspectives. Dublin: University College Dublin Press; 2006.

11. Wrather JA, Koenning SR: Estimates of disease effects on soybean yields in the United States 2003 to 2005. Journal of nematology 2006, 38(2):173-180

12. Tyler BM, Tripathy S, Zhang $X$, Dehal $P$, Jiang $R H$, Aerts $A$, Arredondo FD, Baxter L, Bensasson D, Beynon JL, et al.: Phytophthora genome sequences uncover evolutionary origins and mechanisms of pathogenesis. Science (New York, NY) 2006, 313(5791):1261-1266.

13. Haas BJ, Kamoun S, Zody MC, Jiang RH, Handsaker RE, Cano LM, Grabher M, Kodira CD, Raffaele S, Torto-Alalibo T, et al.: Genome sequence and analysis of the Irish potato famine pathogen Phytophthora infestans. Nature 2009.
14. Govers F, Meijer HJ: Phytophthora-genomics: nieuwe mogelijkheden en uitdagingen. Gewasbescherming 2007, 38(5):265-271.

15. Jiang RH, Govers F: Nonneutral GC3 and retroelement codon mimicry in Phytophthora. Journal of molecular evolution 2006, 63(4):458-472.

16. Judelson HS: Sequence variation and genomic amplification of a family of Gypsy-like elements in the oomycete genus Phytophthora. Mol Biol Evol 2002, 19(8):1313-1322.

17. Ah Fong AM, Judelson HS: The hAT -like DNA transposon DodoPi resides in a cluster of retro- and DNA transposons in the stramenopile Phytophthora infestans. Molecular genetics and genomics 2004, 271(5):577-585

18. Jiang RH, Dawe AL, Weide R, van Staveren M, Peters S, Nuss DL, Govers F: Elicitin genes in Phytophthora infestans are clustered and interspersed with various transposon-like elements. Molecular genetics and genomics 2005, 273(1):20-32.

19. Martens C, Vandepoele $K$, Van de Peer Y: Whole-genome analysis reveals molecular innovations and evolutionary transitions in chromalveolate species. Proc Natl Acad Sci USA 2008, 105(9):3427-3432.

20. Aury JM, Jaillon O, Duret L, Noel B, Jubin C, Porcel BM, Segurens B, Daubin $V$, Anthouard $V$, Aiach $N$, et al.: Global trends of whole-genome duplications revealed by the ciliate Paramecium tetraurelia. Nature 2006, 444(7116):171-178

21. Eisen JA, Coyne RS, Wu M, Wu D, Thiagarajan M, Wortman JR, Badger JH, Ren $\mathrm{Q}$, Amedeo $\mathrm{P}$, Jones KM, et al:: Macronuclear genome sequence of the ciliate Tetrahymena thermophila, a model eukaryote. PLoS Biol 2006, 4(9):e286

22. Jiang RH, Tyler BM, Govers F: Comparative analysis of Phytophthora genes encoding secreted proteins reveals conserved synteny and lineage-specific gene duplications and deletions. Molecular plantmicrobe interactions 2006, 19(12):1311-1321.

23. Van de Peer Y: Computational approaches to unveiling ancient genome duplications. Nature reviews 2004, 5(10):752-763.

24. Simillion C, Vandepoele K, Saeys Y, Van de Peer Y: Building genomic profiles for uncovering segmental homology in the twilight zone. Genome Res 2004, 14(6):1095-1106.

25. Simillion C, Janssens K, Sterck L, Van de Peer Y: i-ADHoRe 2.0: an improved tool to detect degenerated genomic homology using genomic profiles. Bioinformatics (Oxford, England) 2008, 24(1):127-128.

26. Tang $H$, Wang $X$, Bowers JE, Ming R, Alam M, Paterson AH: Unraveling ancient hexaploidy through multiply-aligned angiosperm gene maps. Genome Res 2008, 18(12):1944-1954.

27. Van de Peer Y, Fawcett JA, Proost S, Sterck L, Vandepoele K: The flowering world: a tale of duplications. Trends in Plant Science 2009, 14(12):680-688

28. Wolfe $\mathrm{KH}$, Shields DC: Molecular evidence for an ancient duplication of the entire yeast genome. Nature 1997, 387(6634):708-713.

29. Kellis M, Birren BW, Lander ES: Proof and evolutionary analysis of ancient genome duplication in the yeast Saccharomyces cerevisiae. Nature 2004, 428(6983):617-624.

30. Dehal P, Boore JL: Two rounds of whole genome duplication in the ancestral vertebrate. PLoS biology 2005, 3(10):e314.

31. Jaillon O, Aury JM, Brunet F, Petit JL, Stange-Thomann N, Mauceli E, Bouneau L, Fischer C, Ozouf-Costaz C, Bernot A, et al.: Genome duplication in the teleost fish Tetraodon nigroviridis reveals the early vertebrate proto-karyotype. Nature 2004, 431(7011):946-957.

32. Vandepoele K, De Vos W, Taylor JS, Meyer A, Van de Peer Y: Major events in the genome evolution of vertebrates: paranome age and size differ considerably between ray-finned fishes and land vertebrates. Proc Natl Acad Sci USA 2004, 101(6):1638-1643

33. Consortium CeS: Genome sequence of the nematode C. elegans: a platform for investigating biology. Science (New York, NY) 1998, 282(5396):2012-2018.

34. Katju V, Lynch M: The structure and early evolution of recently arisen gene duplicates in the Caenorhabditis elegans genome. Genetics 2003, 165(4):1793-1803.

35. Hillier LW, Coulson A, Murray Jl, Bao Z, Sulston JE, Waterston RH Genomics in C. elegans: so many genes, such a little worm. Genome Res 2005, 15(12):1651-1660

36. Vergara IA, Mah AK, Huang JC, Tarailo-Graovac M, Johnsen RC, Baillie DL, Chen N: Polymorphic segmental duplication in the nematode Caenorhabditis elegans. BMC genomics 2009, 10:329. 
37. Mok BW, Ribacke U, Sherwood E, Wahlgren M: A highly conserved segmental duplication in the subtelomeres of Plasmodium falciparum chromosomes varies in copy number. Malaria journal 2008, 7:46.

38. Dujon B, Sherman D, Fischer G, Durrens P, Casaregola S, Lafontaine I, De Montigny J, Marck C, Neuveglise C, Talla E, et al.: Genome evolution in yeasts. Nature 2004, 430(6995):35-44.

39. Fairhead C, Dujon B: Structure of Kluyveromyces lactis subtelomeres: duplications and gene content. FEMS yeast research 2006, 6(3):428-441

40. Van de Peer Y, Maere S, Meyer A: The evolutionary significance of ancient genome duplications. Nature Reviews Genetics 2009, 10(10):725-732

41. Nei M, Kumar S: Molecular Evolution and Phylogenetics. New York: Oxford Univ. Press; 2000

42. Hughes AL: Adaptive Evolution of Genes and Genomes. New York: Oxford Univ. Press; 1999

43. Kroon LP, Bakker FT, van den Bosch GB, Bonants PJ, Flier WG Phylogenetic analysis of Phytophthora species based on mitochondrial and nuclear DNA sequences. Fungal Genet Biol 2004, 41(8):766-782.

44. Cavalcanti AR, Ferreira R, Gu Z, Li WH: Patterns of gene duplication in Saccharomyces cerevisiae and Caenorhabditis elegans. J Mol Evol 2003, 56(1):28-37

45. Kappe SH, Noe AR, Fraser TS, Blair PL, Adams JH: A family of chimeric erythrocyte binding proteins of malaria parasites. Proc Natl Acad SCi USA 1998, 95(3):1230-1235.

46. Morris PF, Phuntumart V: Inventory and comparative evolution of the $A B C$ superfamily in the genomes of Phytophthora ramorum and Phytophthora sojae. Journal of molecular evolution 2009, 68(5):563-575.

47. Qutob D, Tedman-Jones J, Dong S, Kuflu K, Pham H, Wang Y, Dou D, Kale $\mathrm{SD}$, Arredondo FD, Tyler BM, et al.: Copy number variation and transcriptional polymorphisms of Phytophthora sojae RXLR effector genes Avr1a and Avr3a. PloS one 2009, 4(4):e5066.

48. Blanc $\mathrm{G}$, Wolfe KH: Functional divergence of duplicated genes formed by polyploidy during Arabidopsis evolution. Plant Cell 2004 16(7):1679-1691.

49. Seoighe C, Gehring C: Genome duplication led to highly selective expansion of the Arabidopsis thaliana proteome. Trends Genet 2004, 20(10):461-464.

50. Birchler JA, Bhadra U, Bhadra MP, Auger DL: Dosage-dependent gene regulation in multicellular eukaryotes: implications for dosage compensation, aneuploid syndromes, and quantitative traits. Dev Biol 2001, 234(2):275-288.

51. Maere S, De Bodt S, Raes J, Casneuf T, Van Montagu M, Kuiper M, Van de Peer Y: Modeling gene and genome duplications in eukaryotes. Proc Natl Acad Sci USA 2005, 102(15):5454-5459.

52. Edger PP, Pires JC: Gene and genome duplications: the impact of dosage-sensitivity on the fate of nuclear genes. Chromosome Res 2009, 17(5):699-717.

53. Freeling M: Bias in plant gene content following different sorts of duplication: tandem, whole-genome, segmental, or by transposition. Annu Rev Plant Biol 2009, 60:433-453.

54. Galiana E, Fourre S, Engler G: Phytophthora parasitica biofilm formation: installation and organization of microcolonies on the surface of a host plant. Environ Microbio/ 2008, 10(8):2164-2171.

55. Irving HR, Griffith JM, Grant BR: Calcium efflux associated with encystment of Phytophthora palmivora zoospores. Cell Calcium 1984, 5(5):487-500.

56. Reid B, Morris BM, Gow NAR: Calcium-dependent, genus-specific, autoaggregation of zoospores of phytopathogenic fungi. Exp Mycol 1995, 19:202-213.

57. Tyler BM: Molecular basis of recognition between phytophthora pathogens and their hosts. Annual review of phytopathology 2002, 40:137-167.

58. Deacon JW, Donaldson SP: Molecular recognition in the homing response of zoosporic fungi, with special reference to Pythium and Phytophthora. Mycol Res 1993, 97:1153-1171.

59. Costanzo S, Ospina-Giraldo MD, Deahl KL, Baker CJ, Jones RW: Gene duplication event in family 12 glycosyl hydrolase from Phytophthora spp. Fungal Genet Biol 2006, 43(10):707-714

60. Seoighe C, Wolfe KH: Updated map of duplicated regions in the yeast genome. Gene 1999, 238(1):253-261.

61. Birchler JA, Riddle NC, Auger DL, Veitia RA: Dosage balance in gene regulation: biological implications. Trends Genet 2005, 21(4):219-226.
62. Freeling M, Thomas BC: Gene-balanced duplications, like tetraploidy, provide predictable drive to increase morphological complexity. Genome Res 2006, 16(7):805-814

63. Birchler JA, Veitia RA: The gene balance hypothesis: from classical genetics to modern genomics. Plant Cell 2007, 19(2):395-402.

64. Birchler JA, Veitia RA: The gene balance hypothesis: implications for gene regulation, quantitative traits and evolution. New Phytol 2009.

65. Sansome E: Polyploidy and Induced Gametangial Formation in Britisch Isolates of Phytophthora infestans. Journal of General Microbiology 1977 , 99:311-316

66. Daggett SS, Knighton JE, Therrien CD: Polyploidy Among Isolates of Phytophthora infestans from Eastern Germany. Journal of Phytopathology 1995, 143(7):419-422.

67. Judelson HS, Yang G: Recombination pathways in Phytophthora infestans: polyploidy resulting from aberrant sexual development and zoospore-mediated heterokaryosis. Mycological Research 1998, 102(10):1245-1253.

68. Sansome ER, Brasier CM, Hamm PB: Phytophthora meadii may be a species hybrid. Mycological Research 1991, 95:273-277.

69. Whittaker SL, Shattock RC, Shaw DS: Variation in DNA content of nuclei of Phytophthora infestans as measured by a microfluorimetric method using fluorochrome DAPI. Mycological Research 1991, 95:602-610.

70. Win-Tin Dick MW: Cytology of Oomycetes. Arch Microbiol 1975, 105:283-293.

71. Malcolmson JF: Races of Phytophthora infestans occurring in Great Britain. Transactions of the British Mycological Society 1969, 53:417-423.

72. Altschul SF, Madden TL, Schaffer AA, Zhang J, Zhang Z, Miller W, Lipman DJ: Gapped BLAST and PSI-BLAST: a new generation of protein database search programs. Nucleic Acids Res 1997, 25(17):3389-3402

73. Enright AJ, Van Dongen S, Ouzounis CA: An efficient algorithm for largescale detection of protein families. Nucleic Acids Res 2002 30(7):1575-1584.

74. Van Dongen S: Graph Clustering by Flow Simulation. In PhD thesis University of Utrecht; 2000

75. Thompson JD, Higgins DG, Gibson TJ: CLUSTAL W: improving the sensitivity of progressive multiple sequence alignment through sequence weighting, position-specific gap penalties and weight matrix choice. Nucleic Acids Res 1994, 22(22):4673-4680.

76. Goldman N, Yang Z: A codon-based model of nucleotide substitution for protein-coding DNA sequences. Mol Biol Evol 1994, 11(5):725-736.

77. Yang Z: PAML: a program package for phylogenetic analysis by maximum likelihood. Comput Appl Biosci 1997, 13(5):555-556.

78. Harris MA, Clark J, Ireland A, Lomax J, Ashburner M, Foulger R, Eilbeck K, Lewis S, Marshall B, Mungall C, et al.: The Gene Ontology (GO) database and informatics resource. Nucleic Acids Res 2004, 32(Database issue):D258-261.

79. Zdobnov EM, Apweiler R: InterProScan--an integration platform for the signature-recognition methods in InterPro. Bioinformatics 2001, 17(9):847-848

80. Storey JD, Tibshirani R: Statistical significance for genomewide studies. Proc Natl Acad Sci USA 2003, 100(16):9440-9445.

doi: $10.1186 / 1471-2164-11-353$

Cite this article as: Martens and Van de Peer, The hidden duplication past of the plant pathogen Phytophthora and its consequences for infection BMC Genomics 2010, 11:353 